\title{
Helminth fauna of the Eurasian Marsh Frog, Pelophylax ridibundus (Pallas, 1771) (Anura: Ranidae), collected from Denizli Province, Inner-West Anatolia Region, Turkey
}

\author{
S. DÜŞEN ${ }^{1 *}, M . ~ O ̈ Z^{2}$ \\ 1*Pamukkale University, Faculty of Arts and Sciences, Department of Biology, Kinikli Campus, 20017, Denizli, \\ Turkey, E-mail: sdusen@pamukkale.edu.tr, serdar2290@yahoo.com; ${ }^{2}$ Akdeniz University Faculty of Science, \\ Department of Biology, 07058, Antalya, Turkey
}

\begin{abstract}
Summary
In this research, a total of 298 Eurasian marsh frogs, Pelophylax ridibundus (Pallas, 1771) were collected from different localities in Denizli province (Inner-west Anatolia Region, the eastern part of Aegean Region) Turkey between 2006 and 2009 and examined for helminths. Of 262 (87.91\%) Pelophylax ridibundus samples were infected with one or more helminths. Pelophylax ridibundus harbored eight species of digeneans (Diplodiscus subclavatus, Gorgoderina vitelliloba, Gorgodera cygnoides, Pleurogenoides medians, Prosotocus confusus, Skrjabinoeces breviansa, Encyclometra colubrimurorum and Ophistoglyhe ranae), one species of cestode (Nematotaenia dispar), two species of acanthocephalans (Acanthocephalus ranae and Pomphorhynchus laevis), and six species of nematodes (Rhabdias bufonis, Oswaldocruzia filiformis, Cosmocerca ornata, Oxysomatium brevicaudatum, Eustrongylides sp. and Abbreviata sp.). P. ridibundus represents a host record for Nematotaenia dispar in Turkey.
\end{abstract}

Keywords: Amphibians, Denizli, Helminth, Pelophylax ridibundus, Turkey

\section{Introduction}

The Eurasian marsh frog, Pelophylax ridibundus (formerly known as Rana ridibunda), is a medium-sized semiaquatic anuran species. $P$. ridibundus inhabits lakes, pools, or slowly flowing streams. It is a highly opportunistic diurnal amphibian, living in mixed and deciduous forests, forest steppe, and steppe and other grasslands, semi-desert and desert zones. Arid areas are largely colonized through river valleys and channels. P. ridibundus prefers open, well-warmed areas with abundant herbaceous vegetation IUCN, 2012). In Turkey, this species is known in all suitable habitats except for a portion of the Lakes District (Baran \& Atatür, 1997; Budak \& Göçmen, 2008).
To our knowledge, the first helminthological study in Eurasian marsh frog, $P$. ridibundus (formerly known as Rana ridibunda) was reported by Schad et al. (1960) and they recorded only two nematodes in this species from Turkey. Other helminthological studies on $P$. ridibundus published by Saygı and Başıüyük (1990), Oğuz et al. (1994), Yildırımhan et al. (1996, 1997, 2005a), Kir et al. (2001), Düşen and Öz (2006), Sağlam and Arıkan (2006), Unal et al. (2007), Düşen and Oğuz (2008, 2010), Düşen et al. (2010a), Heckmann et al. $(2010,2011)$ in Turkey.

So far, there has been no published detailed study on helminths of Eurasian marsh frog, $P$. ridibundus from Denizli province (Inner-west Anatolia Region - the eastern part of Aegean Region) in Turkey. This is the first detailed helminthological study, which has been done in this geographic area.

\section{Materials and methods}

Frog samples were collected by hand and dip net, between 2006 - 2009 from 17 different localities in Denizli province $\left(38^{\circ} 29^{\prime}-38^{\circ} 52^{\prime} \mathrm{N}-28^{\circ} 38^{\prime}-30^{\circ} 05^{\prime} \mathrm{E}\right)$. In total,

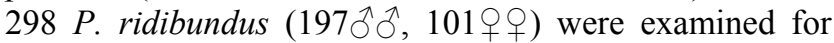
helminth parasites. The mean \pm SD snout-vent length (SVL) of specimens was $66.34 \pm 14.91 \mathrm{~mm}$, with a range from 29 to $101 \mathrm{~mm}$; within $24 \mathrm{hr}$, toads were overdosed in ether-filled glass containers.

The samples body cavity was opened by a longitidutinal ventral incision. The alimentary canal was excised and separated into stomach, small intestine, large intestine and rectum. The contents of each part and other organs (lungs, liver, gall bladder, kidneys and urinary bladder) were each mixed with $0.5 \%$ saline solution and poured into petri dishes for examination under a stereomicroscope. The muscles, plus portions of peritoneum and spinal cord, were teased out with needles and examined under a stereomicro- 
scope. Digeneans was immobilized by coverslip pressure in $70 \%$ ethyl alcohol, fixed, and stored in $70 \%$ ethanol. Nematodes was straightened by heat, fixed, and stored in $70 \%$ ethyl alcohol with $5 \%$ glycerol. Acanthocephalans were relaxed in saline and heat-fixed under slight coverslip pressure in warm ethyl alcohol-formalin-acetic acid. Digeneans, acanthocephalans and cestode samples were stained with acetocarmine, dehydrated, cleared in cedar oil or xylol, and mounted in Canada Balsam; nematodes were cleared in glycerol and examined. Intensities are presented as mean values $( \pm 1 \mathrm{SE})$ followed by the range. Voucher host specimens and parasite specimens were deposited in Pamukkale University, Faculty of Sciences and Arts, Department of Biology, Denizli, Turkey (PAU-HELM-117/2009).

\section{Results and discussion}

Two-hundred and ninety eight $P$. ridibundus (197ठ̂̉, 101 웅) samples were collected between 2006 - 2009 years from Denizli province.

\section{Trematoda}

Family: Diplodiscidae

Diplodiscus subclavatus (Pallas, 1760) Diesing, 1836

Prevalence, intensity and range: Hosts infected, 31 of 298 $(10.40 \%, 4.03 \pm 0.65 \mathrm{SE}, 1-15)$.

Other reported hosts: D. subclavatus was observed in, Triturus cristatus, T. vulgaris, Bufo viridis, Hyla arborea (Shimalov et al., 2001; Vojtková \& Vojtek 1975, Buchvarov 1977, Kuc \& Sulgostowska 1988b, Sey 1991); H. savignyi (Yıldırımhan et al., 2012); Rana temporaria, R. dalmatina, $R$. esculenta, R. graeca (Vojtková \& Vojtek 1975; Buchvarov, 1977; Kuc \& Sulgostowska, 1988b; Sey, 1991; Düşen et al., 2009), Bombina bombina, Bo. variegata (Vojtková \& Vojtek, 1975; Grabda-Kazubska \& Lewin, 1989), Pelobates syriacus balcanicus (Buchvarov, 1977), T. alpestris, P. fuscus, R. lessonae (Vojtková \& Vojtek, 1975), B. bufo, R. arvalis (Vojtková \& Vojtek, 1975; Sey 1991; Shimalov \& Shimalov, 2001), R. holtzi (Topçu, 2002; Y1ldırımhan et al., 2006b), Natrix natrix (Sey, 1991; Buchvarov et al., 2000; Shimalov \& Shimalov, 2000), N. tessellata (Buchvarov et al., 2000), Vipera berus (Shimalov \& Shimalov, 2000) and Esox lucius (Öztürk et al., 2000).

Geographic range: Europe (Yamaguti, 1958), Czech Republic (Vojtková \& Vojtek, 1975), Bulgaria (Buchvarov et al., 1975; Buchvarov, 1977), Poland (Kuc \& Sulgostowska, 1988a, 1988b; Grabda-Kazubska \& Lewin, 1989), Hungaria (Sey, 1991), Turkey (Oğuz et al., 1994), Iran (Masshaii et al., 2000), Uzbekistan (Vashetko \& Siddikov, 1999).

Specimens deposited: PAU-HELM-1/2009

Family: Gorgoderidae

Gorgoderina vitelliloba (Olsson, 1876) Loos, 1902

Prevalence, intensity and range: Hosts infected, 20 of 298 $(6.71 \%, 2.55 \pm 0.81 \mathrm{SE}, 1-13)$.

Other reported hosts: G. vitelliloba was reported in, Bombinator igneus (Yamaguti, 1958), Bo. bombina (Vojtková
\& Vojtek, 1975), Bo. variegata, (Yamaguti, 1958; Vojtková \& Vojtek, 1975; Buchvarov, 1977) B. bufo and B. calamita (Cedhagen, 1988), B. viridis (Buchvarov, 1977), $R$. arvalis (Yamaguti, 1958; Vojtková \& Vojtek, 1975), $R$. dalmatina (Buchvarov, 1977), R. esculenta, (Vojtková \& Vojtek, 1975), R. temporaria (Yamaguti, 1958; Vojtková \& Vojtek, 1975; Buchvarov, 1977; Cedhagen, 1988), $R$. macrocnemis (Yıldırımhan et al., 1997b; Y1ldırımhan et al., 2006a), R. camerani (Y1ldırımhan et al., 2006a) and $R$. holtzi (Y1ldirımhan et al., 2006b).

Geographic range: Europe and North America (Yamaguti, 1958), Czech Republic (Vojtková \& Vojtek, 1975), Bulgaria (Buchvarov, 1977), Poland (Kuc \& Sulgostowska, 1988a, 1988b), Turkey (Yıldırımhan et al., 1996; Düşen \& Öz, 2006).

Specimens deposited: PAU-HELM-2/2009

\section{Family: Gorgoderidae}

Gorgodera cygnoides (Zeder, 1800)

Prevalence, intensity and range: Hosts infected, 36 of 298 (12.08\%, $3.83 \pm 0.70 \mathrm{SE}, 1-17)$.

Other reported hosts: G. cygnoides is a common parasite of amphibians, Bombinator igneus (Yamaguti, 1958), Bo. Bombina and Bo. variegata (Vojtková \& Vojtek, 1975), B. viridis (Buchvarov, 1977; Shimalov \& Shimalov, 2001), $H$. arborea (Yamaguti, 1958), R. arvalis (Vojtková \& Vojtek, 1975), R. dalmatina (Buchvarov, 1983), R. esculenta (Yamaguti, 1958; Vojtková \& Vojtek, 1975; Buchvarov, 1977; Kuc \& Sulgostowska, 1988b), R. lessonae (Vojtková \& Vojtek, 1975), R. clamitans (Yamaguti, 1958), R. temporaria (Yamaguti, 1958; Vojtková \& Vojtek, 1975), $R$. camerani (Yıldırımhan et al., 2006a), R. holtzi (Yıldırımhan et al., 2006b), R. kurtmuelleri (Hristovski et al., 2006) and Pelodytes caucasicus (Y1ldirimhan et al., 2009).

Geographic range: Western Europe (Yamaguti, 1958) Turkey (Yıldırımhan et al., 1996; Düşen \& Öz, 2006).

Specimens deposited: PAU-HELM-3/2009

\section{Family: Lecithodendriidae}

\section{Pleurogenoides medians (Olsson, 1876) Travassos, 1921}

Prevalence, intensity and range: Hosts infected, 47 of 298 (15.77\%, 27.70 \pm 4.57 SE, 1 - 126).

Other reported hosts: $P$. medians has been reported in various amphibians and reptiles species, T. cristatus (Shimalov et al., 2001), T. vulgaris (Vojtková \& Vojtek, 1975; Shimalov et al., 2001); Bo. bombina, (Vojtková \& Vojtek, 1975), Bo. variegata, (Vojtková \& Vojtek, 1975), B. bufo (Shimalov\& Shimalov, 2001), B. calamita, (Vojtková \& Vojtek, 1975), B. vulgaris (Yamaguti, 1958), B. viridis (Düşen et al., 2010; Düşen \& Oğuz, 2010), H. arborea (Vojtková \& Vojtek, 1975; Düşen \& Öz, 2004), H. savignyi (Y1ldırımhan et al., 2012), R. arvalis (Vojtková \& Vojtek, 1975), R. camerani (Y1ldırımhan et al., 2006a; Düşen, 2007), R. dalmatina (Buchvarov, 1977; Düşen et al., 2009), R. esculenta (Vojtková \& Vojtek, 1975; Buchvarov, 1977; Kuc \& Sulgostowska, 1988b), R. macrocnemis (Yıldırımhan et al., 2006b; Düşen, 2007), R. arvalis (Vojtková \& Vojtek, 1975), R. temporaria (Vojtková \& 
Vojtek, 1975; Cedhagen, 1977), R. holtzi (Topçu, 2002), Lacerta trilineata (Yamaguti, 1963; Yildırımhan et al., 2011), N. natrix (Kirin, 2000b) and Atheris hispida (Hassl, 2010; Hassl et al., 2010).

Geographic range: Europe and Asia (Yamaguti, 1958); Austrolasian Regions (Prudhoe \& Bray, 1982).

Specimens deposited: PAU-HELM-4/2009

Family: Lecithodendriidae

Prosotocus confusus (Loos, 1894) Loos, 1899

Prevalence, intensity and range: Hosts infected, 23 of 298 (7.72\%, $5.82 \pm 1.33 \mathrm{SE}, 1-33)$.

Other reported hosts: $R$. esculenta (Yamaguti, 1958; Vojtková \& Vojtek, 1975; Buchvarov, 1977; Kuc \& Sulgostowska, 1988b), B. viridis (Yamaguti, 1958; Vojtková \& Vojtek, 1975), B. vulgaris (Yamaguti, 1958), and Bo. variegata, B. bufo, B. calamita, H. arborea, $R$. temporaria, $R$. arvalis (Vojtková \& Vojtek, 1975; Buchvarov, 1977), This species is only observed in $R$. ridibunda (Yıldırımhan et al., 1996, 2005a; Düşen \& Öz 2006) from Turkey. Geographic range: Europe and Asia (Yamaguti, 1958), Turkey (Y1ldırımhan et al., 1996, 2005a; Düşen \& Öz 2006).

Specimens deposited: PAU-HELM-5/2009

Family: Plagiorchiidae

Skrjabinoeces breviansa (Sudarikov, 1950)

Prevalence, intensity and range: Hosts infected, 29 of 298 $(9.73 \%, 5.34 \pm 1.10 \mathrm{SE}, 1-35)$.

Other reported hosts: Sey and Eory (1992) were reported Skrjabinoeces breviansa in $R$. ridibunda from Hungary, Mashaii et al. (2000) were reported S. breviansa in $R$. ridibunda from Northern parts of Iran. Kovalenko (2007) was recorded this digenean in $R$. ridibunda and $R$. esculenta from Ukrain. Yıldırımhan et al. (2005a), Düşen and Öz (2006), Düşen and Oğuz (2010) were recorded this helminth species in $R$. ridibunda from Turkey.

Geographic range: Europe, Russia (Yamaguti, 1958), Turkey (Yıldırımhan et al., 2005a; Düşen and Öz (2006), and Iran (Mashaii et al., 2000).

Specimens deposited: PAU-HELM-6/2009

Family: Plagiorchiidae

Encyclometra colubrimurorum (Rudolphi, 1819) Dollfus, 1922 (Metacercariae)

Prevalence, intensity and range: Hosts infected, 7 of 298 $(2.35 \%, 2.43 \pm 1.11 \mathrm{SE}, 1-8)$.

Other reported hosts: $R$. tigrina, Xenochnopis piscator, Coluber gemonensis, Ptyas mucosus, (Yamaguti, 1958); N. natrix (Yamaguti, 1958; Shimalov \& Shimalov, 2000; Mihalca et al., 2007); Bo. bombina, P. fuscus, B. viridis, $R$. dalmatina, $R$. temporaria (Vojtková \& Vojtek, 1975); $R$. esculenta (Buchvarov, 1977); H. arborea (Vojtková \& Vojtek, 1975; Düşen \& Öz, 2004). The definitive host of E. colubrimurorum is a colubrid snake, N. natrix (grass snake) (Liang-Sheng, 1958), E. colubrimurorum parasitised $P$. ridibundus as an intermediate host. Düşen and Öz (2006) observed this digenean from $R$. ridibunda in An- talya region in Turkey.

Geographic range: Europe, Asia (Yamaguti, 1958); Czech Republic, France, Italy, Russia, The Netherlands (Vojtková \& Vojtek, 1975); Iran (Masshaii et al., 2000); Turkey (Düşen \& Öz, 2004; Düşen \& Öz, 2006).

Specimens deposited: PAU-HELM-7/2009

Family: Telorchiidae

Opisthioglyphe ranae (Froelich, 1791)

Prevalence, intensity and range: Hosts infected, 4 of 298 $(1.34 \%, 4.75 \pm 0.85 \mathrm{SE}, 3-7)$.

Other reported hosts: The reported hosts of $O$. ranae were, Salamandra maculosa (Vojtková \& Vojtek, 1975; Buchvarov, 1977); T. cristatus (Yamaguti, 1958; Shimalov et al., 2001), T. vulgaris (Shimalov et al., 2001), Bo. bombina (Vojtková \& Vojtek, 1975; Y1ldırımhan et al., 2001a), Bo. variegata (Vojtková \& Vojtek, 1975), B. bufo (Shimalov \& Shimalov, 2001), B. calamita (Yamaguti, 1958; Shimalov \& Shimalov, 2001), B. vulgaris, B. variabilis, (Yamaguti, 1958), B. viridis, (Vojtková \& Vojtek, 1975; Buchvarov, 1977), H. arborea (Vojtková \& Vojtek, 1975; Buchvarov, 1977 ), P. fuscus (Vojtková \& Vojtek, 1975); R. dalmatina (Vojtková \& Vojtek, 1975; Buchvarov, 1977), R. esculenta (Yamaguti, 1958; Vojtková \& Vojtek, 1975; Buchvarov, 1977), R. lessonae (Vojtková \& Vojtek, 1975), R. temporaria (Yamaguti, 1958; Vojtková \& Vojtek, 1975; Buchvarov, 1977), R. arvalis (Vojtková \& Vojtek, 1975), $R$. camerani (Yıldırımhan et al., 2006a), R. holtzi (Topçu, 2002), N. Natrix and V. berus (Shimalov \& Shimalov, 2000).

Geographic range: Europe (Yamaguti, 1958), Turkey (Yıldırımhan et al., 1996; Düşen \& Öz, 2006), Saudi Arabia (Fernando, 1989), Iran (Masshaii et al., 2000).

Specimens deposited: PAU-HELM-8/2009

\section{Cestoda}

Family: Nematotaeniidae

Nematotaenia dispar (Goeze, 1782) Lühe, 1899

Prevalence, intensity and range: 2 of 298 hosts infected $(0.67 \%, 2,2)$.

Other reported hosts: $N$. dispar is commonly parasitic in the intestines of amphibians, but rarely reptiles of the Oriental, Nearctic, and Palearctic regions (Prudhoe \& Bray, 1982), including Bo. bombina (Buchvarov, 1977), Bo. variegata (Buchvarov, 1977; Prudhoe \& Bray, 1982), B. bufo (Hristovski \& Riggio, 1971; Prudhoe \& Bray, 1982), B. alvarius (Goldberg \& Bursey, 1991), H. arborea (Hristovski \& Riggio, 1974; Buchvarov, 1977; Prudhoe \& Bray, 1982; Vashetko \& Siddikkov, 1999; Saeed et al., 2007), H. savignyi (Al-Sorkhy \& Amr, 2003; Mashaii, 2005; Yildırımhan et al. 2012), R. temporaria (Hristovski \& Lees, 1973, Buchvarov, 1997), R. dalmatina (Buchvarov, 1977), Mertensiella caucasica (Y1ldırımhan et al., 2001b; 2005b), Amietophrynus (Bufo) regularis (Ibrahim, 2008). B. viridis (Hristovski \& Riggio, 1971; Buchvarov, 1977; Y1ldırımhan, 1999; Düşen, 2003, 2011; Saeed et al., 2007; Masshaii et al., 2008; Düşen et al., 2010a; Düşen \& Oğuz, 
2010), R. camerani (Yıldırımhan et al., 2006a). Buchvarov (1977) recorded this cestode in $R$. ridibunda from, Bulgaria.

Geographic range: Palaearctic, Oriental and Australian regions (Prudhoe \& Bray, 1982; Al-Sorkhy \& Amr, 2003). Nematotaenia dispar is first time observed in P. ridibundus from Turkey.

Specimens deposited: PAU-HELM-9/2009

\section{Nematoda}

Family: Rhabdiasidae

Rhabdias bufonis (Schrank, 1788) Stiles and Hassal, 1905 Prevalence, intensity and range: 19 of 298 hosts infected $(6.37 \%, 2.05 \pm .0 .23 \mathrm{SE}, 1-10)$.

Other reported hosts: $R$. bufonis is known from various amphibian species including, Bufo sp., Rana sp., Pelobates sp., Bombinator sp., Anguis fragilis (Yamaguti, 1961; Düşen et al., 2010b), Bo. bombina (Grabda-Kazubska \& Lewin, 1989; Y1ldırımhan et al., 2001a), Pe. caucasicus (Yildırımhan et al., 2009), R. esculenta (Buchvarov, 1977; Kuc \& Sulgostowska, 1988b), Bo. variegata, P. syriacus (Buchvarov, 1977), B. viridis (Buchvarov et al., 1975; Buchvarov, 1977; Yildırımhan, 1999a; Shimalov \& Shimalov, 2001; Düşen, 2011), B. bufo (Buchvarov, 1977; Shimalov \& Shimalov, 2001; Y1ldırımhan \& Karadeniz, 2007; Düşen, 2011), B. calamita (Shimalov \& Shimalov, 2001), Amietophrynus (Bufo) regularis (Ibrahim, 2008), $R$. temporaria, $R$. arvalis (Kuc \& Sulgostowska, 1988b; Cedhagen, 1988), R. dalmatina (Buchvarov et al., 1975; Buchvarov, 1977; Düşen et al., 2009), R. camerani (Yildırımhan et al., 2006a), R. macrocnemis (Yıldırımhan et al., 2006b).

Geographic range: Europe, Siberia, China, Canada, U.S.A. (Yamaguti, 1961).

Specimens deposited: PAU HEL-10/2009

Family: Molineidae

Oswaldocruzia filiformis (Goeze, 1782) Travassos, 1917

Prevalence, intensity and range: 17 of 298 hosts infected (5.70 \%, $2.47 \pm 0.70 \mathrm{SE}, 1-10)$.

Other reported hosts: O. filiformis is recorded from various amphibian and reptile species, including Salamandra salamandra (Buchvarov, 1977), T. alpestris and T. karelinii (Buchvarov, 1977; Cedhagen 1988; Kirin \& Buchvarov, 2002), T. vulgaris (Buchvarov, 1977; Shimalov et al., 2001), T. vittatus (Y1ldırımhan, 2008), Bo. bombina and Bo. variegata (Buchvarov, 1977; Kirin \& Buchvarov, 2002), B. regularis (probably Bufo viridis) (Schad et al., 1960), B. viridis (Buchvarov, 1977; Yıldırımhan, 1999; Shimalov \& Shimalov, 2001; Topçu \& Bayrak, 2000; Düşen et al., 2010a; Düşen \& Oğuz, 2010), B. bufo (Buchvarov, 1977; Yıldırımhan \& Karadeniz, 2007; Düşen \& Oğuz, 2010; Düşen, 2011); Pe. caucasicus (Yıldırımhan et al., 2009), H. arborea (Buchvarov, 1977; Yildırımhan et al., 2006c), R. camerani (Yıldırımhan et al., 2006a); $H$. savignyi (Y1ldırımhan et al., 2012); R. dalmatina, (Buchvarov et al., 1975; Buchvarov, 1977; Kirin \& Buchvarov, 2002; Düşen et al., 2009), R. kurtmuelleri (Hristovski et al., 2006), R. macrocnemis (Schad et al., 1960; Y1ldırımhan et al., 1997b; Y1ldırımhan et al., 2006b), R. temporaria, (Buchvarov, 1977; Cedhagen, 1988; Kirin \& Buchvarov, 2002), R. graeca (Božkov \& Stojkova, 1970; Buchvarov, 1977), L. agilis, (Sharpilo et al., 2001; Shimalov et al., 2000; Mihalca et al., 2007), L. trilineata (Yildırımhan et al., 2011), L. viridis (Biserkov \& Kostadinova, 1998; Y11dırımhan, 1999b; Kirin, 2002a; Borkovcová \& Kopřiva, 2005), L. vivipara (Shimalov et al., 2000), A. fragilis (Schad et al., 1960; Bertman \& Okulewicz, 1987; Shimalov et al., 2000; Borkovcová \& Kopřiva 2005; Düşen et al, 2010b), Zootoca vivipara (Sanchis et al., 2000), N. natrix (Bertman \& Okulewicz, 1987; Shimalov \& Shimalov, 2000; Kirin 2002b) and V. berus (Shimalov \& Shimalov, 2000).

Geographic range: Europe and Asia (Yamaguti, 1961).

Specimens deposited: PAU-HELM-11/2009

\section{Family: Cosmocercidae}

\section{Cosmocerca ornata (Dujardin, 1845)}

Prevalence, intensity and range: 114 of 298 hosts were infected $(38.25 \%, 5.28 \pm 0.75 \mathrm{SE}, 1-47)$.

Other reported hosts: There are several papers reporting $C$. ornata from many species of amphibians and reptiles, including Bufo, Hyla, Rana, Triturus (Yamaguti, 1961), T. alpestris (Walton, 1933; Buchvarov, 1977; Shimalov et al., 2000), T. cristatus (Walton, 1933; Shimalov et al., 2001), T. vulgaris (Shimalov et al., 2001), Bo. bombina, (Buchvarov, 1977; Grabda-Kazubska \& Lewin, 1989); Bo. variegata (Buchvarov, 1977; Grabda-Kazubska \& Lewin, 1989; Kirin \& Buchvarov, 2002), B. viridis (Schad et al. 1960; Buchvarov et al., 1975; Buchvarov, 1977; Vashetko \& Siddikov,1999; Masshaii, 2005; Düşen \& Oğuz, 2010; Düşen et al., 2010; Düşen 2011), B. bufo (Buchvarov, 1977; Shimalov \& Shimalov, 2001; Yıldırımhan \& Karadeniz, 2007; Düşen \& Oğuz, 2010; Düşen et al., 2010; Düşen 2011); Pe. caucasicus (Yıldırımhan et al., 2009), H. arborea (Buchvarov, 1977, Y1ldırımhan et al., 2006c), $P$. syriacus (Buchvarov, 1977; Shimalov et al., 2000), R. esculenta (Walton, 1933; Buchvarov, 1977), R. arvalis (Cedhagen, 1988; Kuc \& Sulgostowska, 1988b), R. temporaria, (Walton, 1933; Buchvarov, 1977; Kuc \& Sulgostowska, 1988b), R. graeca (Božkov \& Stojkova, 1970; Buchvarov, 1977), R. holtzi (Y1ldırımhan et al., 2006b), $R$. macrocnemis (Schad et al. 1960; Y1ldırımhan et al., 2006b; Düşen, 2007), R. camerani (Yıldırımhan et al., 2006a; Düşen, 2007), R. dalmatina (Buchvarov, 1977; Düşen et al., 2009), R. tavasensis (Düşen, 2012); Chiasmocleis capixaba (Van Sluys et al., 2006) and A. fragilis (Shimalov et al., 2000; Düşen et al., 2010b).

Geographic range: New and Old Worlds (Baker, 1987).

Specimens deposited: PAU-HELM-12/2009

Oxysomatium brevicaudatum (Zeder, 1800) Railliet and Henry, 1916

Prevalence, intensity and range: 22 of 298 hosts were infected $(7.38 \%, 8.86 \pm 2.19 \mathrm{SE}, 1-32)$.

Other reported hosts: $O$. brevicaudatum was reported from 
different hosts of amphibians and reptiles in Europe and Asia, including Bombina, Bufo, Hyla, Pelobates, Rana, Salamandra (Yamaguti, 1961), S. salamandra, S. atra, Pseudotriton ruber and Bo. bombina (Walton, 1933), T. vittatus, T. karelinii (Yıldırımhan, 2008), P. syriacus (Yıldırımhan et al., 1997a; Yildırımhan \& Bursey, 2010), B. viridis (Walton, 1933; Schad et al., 1960; Buchvarov, 1977; Yıldırımhan, 1999a; Topçu \& Bayrak, 2000; Düşen
\& Oğuz, 2010; Düşen et al., 2010; Düşen, 2011), B. bufo (Düşen \& Oğuz, 2010; Düşen et al., 2010; Düşen, 2011), $B$. regularis (probably B. viridis) (Schad et al.,1960), $H$. arborea (Walton, 1933), R. dalmatina (Buchvarov, 1977; Düşen et al., 2009), R. graeca (Božkov \& Stojkova, 1970; Buchvarov, 1977), R. esculenta (Walton, 1933), $R$. kurtmuelleri (Hristovski et al., 2006), R. macrocnemis (Schad et al., 1960), R. temporaria (Walton, 1933; Buch-

Table 1. Prevalence, intensity, infection sites and range of helminths in P. ridibundus

\begin{tabular}{|c|c|c|c|c|c|}
\hline Identified helminth group & $\begin{array}{l}\text { Developmental } \\
\text { stage }\end{array}$ & $\begin{array}{c}\text { Site of } \\
\text { infection }\end{array}$ & $\begin{array}{c}\text { No. of } \\
\text { infected } \\
(\%)\end{array}$ & $\begin{array}{c}\text { Mean } \\
\text { intensity } \\
( \pm \text { SE })\end{array}$ & Range \\
\hline $\begin{array}{l}\text { Diplodiscus subclavatus (Pallas, 1760) } \\
\text { Diesing, } 1836\end{array}$ & Adult & LI & $31(10.40)$ & $4.03 \pm 0.65$ & $1-15$ \\
\hline $\begin{array}{l}\text { Gorgoderina vitelliloba (Olsson, 1876) Loos, } \\
1902\end{array}$ & Adult & UB & $20(6.71)$ & $2.55 \pm 0.81$ & $1-13$ \\
\hline Gorgodera cygnoides (Zeder, 1800) & Adult & UB & $36(12.08)$ & $3.83 \pm 0.70$ & $1-17$ \\
\hline $\begin{array}{l}\text { Pleurogenoides medians (Olsson, 1876) } \\
\text { Travassos, } 1921\end{array}$ & Adult & SI & 47 (15.77) & $27.70 \pm 4.57$ & $1-126$ \\
\hline Prosotocus confusus (Loos, 1894) Loos, 1899 & Adult & SI & $23(7.72)$ & $5.82 \pm 1.33$ & $1-33$ \\
\hline Skrjabinoces breviansa (Sudarikov, 1950) & Adult & $\mathrm{L}$ & $29(9.73)$ & $5.34 \pm 1.10$ & $1-35$ \\
\hline $\begin{array}{l}\text { Encyclometra colubrimurorum } \\
\text { (Rudolphi,1819) Dollfus, } 1922\end{array}$ & Metacercariae & $\mathrm{M}, \mathrm{OS}, \mathrm{BC}$ & $7(2.35)$ & $2.43 \pm 1.11$ & $1-8$ \\
\hline Opisthioglyphe ranae (Froelich, 1791) & Adult & SI & $4(1.34)$ & $4.75 \pm 0.85$ & $3-7$ \\
\hline $\begin{array}{l}\text { Nematotaenia dispar (Goeze, 1782) Lühe, } \\
1899\end{array}$ & Adult & SI & $2(0.67)$ & 2 & 2 \\
\hline $\begin{array}{l}\text { Rhabdias bufonis (Schrank, 1788) Stiles and } \\
\text { Hassal, } 1905\end{array}$ & Adult & $\mathrm{L}$ & $19(6.37)$ & $2.05 \pm 0.23$ & $1-10$ \\
\hline $\begin{array}{l}\text { Oswaldocruzia filiformis (Goeze, 1782) } \\
\text { Travassos, } 1917\end{array}$ & Adult & SI & $17(5.70)$ & $2.47 \pm 0.70$ & $1-10$ \\
\hline Cosmocerca ornata (Dujardin, 1845) & Adult & LI, SI & $114(38.25)$ & $5.28 \pm 0.75$ & $1-47$ \\
\hline $\begin{array}{l}\text { Oxysomatium brevicaudatum (Zeder, 1800) } \\
\text { Railliet and Henry, } 1916\end{array}$ & Adult & LI, SI & $22(7.38)$ & $8.86 \pm 2.19$ & $1-32$ \\
\hline Eustrongylides sp. & Larvae & LI, SI & $3(1.01)$ & 1 & 1 \\
\hline $\begin{array}{l}\text { Abbreviata sp. (Encapsulated larvae in } \\
\text { submucosa of stomach and small intestine) }\end{array}$ & Larvae & $\mathrm{S}, \mathrm{LI}, \mathrm{OS}$ & $35(11.74)$ & 1 & Uncount. \\
\hline $\begin{array}{l}\text { Acanthocephalus ranae (Schrank, 1788) } \\
\text { Lühe, } 1911\end{array}$ & Adult & SI, LI & $63(21.14)$ & $4.92 \pm 0.67$ & $1-26$ \\
\hline $\begin{array}{l}\text { Pomphorhynchus laevis (Zoega in Müller } \\
\text { 1776) }\end{array}$ & Larvae and cysts & M, B, LI, SI & $55(18.45)$ & $21.36 \pm 1.33$ & $1-79$ \\
\hline
\end{tabular}

BC: Body cavities, L: Lung, LI: Large intestine, M: Mesentery, OS: Organ surfaces, R: Rectum, S: Stomach, SI: Small intestine; 
varov, 1977; Kirin \& Buchvarov, 2002), P. fuscus (Walton, 1933), R. kurtmuelleri (Hristovski et al., 2006), A. fragilis (Schad et al., 1960; Sharpilo, 2003; Düşen et al., 2010b), N. natrix (Schad et al., 1960).

Geographic range: Europe and Asia (Yamaguti, 1961). Schad et al. (1960) were first time reported $O$. brevicaudatum in Bufo regularis (probably $B$. viridis), $B$. viridis, $R$. macrocnemis, $R$. ridibunda and $A$. fragilis from Turkey. Sharpilo (2003) pointed out that O. brevicaudatum has a great disperse in $A$. fragilis in the Caucasian Region. Specimens deposited: PAU-HELM-13/2009

Family: Dioctophymatidae

Eustrongylides sp. (larvae)

Prevalence, intensity and range: Hosts infected, 3 of 298 $(1.01 \%, 1,1)$.

Other reported hosts: Eustrongylides sp. is parasitic in glands of proventriculus of aquatic birds. Larvae normally inhabited connective tissue and body cavities of fishes (Yamaguti, 1961). Yildırımhan et al. (1996b) and Aydoğdu et al. (1996a, 1996b, 1997 and 2000) recorded this nematode larvae in N. natrix, roach (Rutilus frisii), sheatfish (Silurus glanis), and tench (Tincta tincta) from Lake Iznik (Bursa). Mihalca et al (2007) were recorded this nematode in N. natrix and N. tesselata in Romania. Anderson (2000) stated that the larvae of this nematode is observed in $R$. ridibunda. Düşen and Öz (2006) reported this nematode larvae in $R$. ridibunda from Antalya Region in Turkey.

Geographic range: Europe south-east Asia, the Middle East and Australia (Anderson, 2000).

Specimens deposited: PAU-HELM-14/2009

Family: Physalopteridae

Abbreviata sp. (larvae - Encapsulated larvae in submucosa of stomach and small intestine).

Prevalence, intensity and range: Hosts infected, 35 of 298 (11.74\%, Uncountable).

Other reported hosts: Fernando (1989) observed this nematode larvae in $R$. ridibunda from Saudi Arabia; Fernando (1989) reported that parasite larvae in brown cysts where located subcutaneously on body wall, heavy infestations also occured in the submucosae of stomach, small intestine and mesenteries and embedded deeply in them muscle. Similiarly, Düşen and Öz (2006) observed this encapsulated larvae in submucosa of stomach and small intestines of $R$. ridibunda from Antalya region in Turkey. Borkovcová and Kopřiva (2005) recorded this nematode species in some lizards (A. fragilis, L. viridis and L. agilis) and one snake (Coronella austriaca) species in South Moravia (Czech Republic). In this study, the larvae of Abbreviata sp. were observed numerous (embedded deeply in submucosa of the stomach and small intestine) therefore counting was difficult.

Geographic range: Europe Asia, and Western Australia (Anderson, 2000).

Specimens deposited: PAU-HELM-15/2009

\section{Acanthocephala}

Family: Echinorhynchidae

Acanthocephalus ranae (Schrank, 1788) Lühe, 1911

Prevalence, intensity and range: 63 of 298 hosts were infected $(21.14 \%, 4.92 \pm 0.67 \mathrm{SE}, 1-26)$.

Other reported hosts: Rana sp., Bombinator sp., Hyla sp., Triturus sp., Salamandra sp., Diemictylus viridescens (Yamaguti, 1963), Bo. bombina (Buchvarov, 1977; Grabda-Kazubska \& Lewin, 1989; Yıldırımhan et al., 2001a), Bo. variegata (Buchvarov, 1977; GrabdaKazubska \& Lewin, 1989), B. viridis (Buchvarov, 1977; Yildırımhan, 1999a; Vashetko \& Siddikov, 1999; Shimalov \& Shimalov, 2001; Düşen \& Oğuz, 2010), B. bufo (Yıldırımhan \& Karadeniz, 2007; Düşen, 2011), B. calamita (Shimalov \& Shimalov, 2001); H. arborea (Düşen \& Öz, 2004), R. arvalis, R. dalmatina (Buchvarov, 1977; Düşen et al, 2009); R. temporaria (Buchvarov, 1977; Cedhagen, 1988; Kuc \& Sulgostowska, 1988b), R. esculenta (Buchvarov, 1977; Kuc \& Sulgostowska, 1988b), $R$. macrocnemis (Yıldırımhan et al., 1997b; Yıldırımhan et al., 2006b; Düşen, 2007), R. camerani (Yıldırımhan et al., 2006a), M. caucasica (Y1ldırımhan et al., 2001b; 2005b), R. kurtmuelleri (Hristovski et al., 2006), R. tavasensis (Düşen, 2012), A. fragilis (Shimalov et al., 2000), N. natrix (Yamaguti, 1963; Shimalov \& Shimalov, 2000).

Geographic range: Europe, U.S.A., Russia (Yamaguti, 1963); Turkey (Oğuz et al., 1994).

Specimens deposited: PAU-HELM-16/2009

Family: Pomphorhynchidae

Pomphorhynchus laevis (Zoega in Müller 1776) (larvae and cysts)

Prevalence, intensity and range: Hosts infected, 55 of 298 $(18.45 \%, 21.36 \pm 1.33 \mathrm{SE}, 1-79)$.

Other reported hosts: In general, Pomphorhynchus laevis occurs predominantly in cyprinid and also in salmonid fishes with the Palaearctic distribution (Dudiňák \& Šnábel, 2001). P. laevis, is observed in several fish species in Turkey: barbel (Barbus plebejus escherichii), bleak (Alburnus alburnus), chub (Leuciscus cephalus), common carp (Cyprinus carpio), crucian carp (Carassius carassius), loach (Nemachilus sp.), nase (Chondrostoma nasus), pike (Esox lucius), sheatfish (Silurus glanis), (Öktener, 2003), and tench (Tinca tinca) (Y1ld1z, 2003). A number of marine and freshwater fishes have been reported as definitive hosts of acanthocephalan P. laevis (Ziolkowska \& Rokicki, 2003). Buchvarov (1977) and Düşen and Oğuz (2008) observed in Rana ridibunda and Veith and Erpelding (1995) in fire salamander ( $S$. salamandra) are reported $P$. laevis.

Geographic distribution: Europe Buchvarov (1977) Specimens deposited: PAU-HELM-17/2009

Seventeen helminth species were found infecting $P$. ridibundus. The site of infection in the Eurasian marsh frog and the data on infection parameters for each host and helminth species are shown in Table 1. According the data 
obtained $262(87.91 \%)$ P. ridibundus harbored the one or more species of helminths and the remaining $36(\%) 12.08$ were uninfected. In summary, 4311 individuals of 17 helminth species were collected from the 298 P. ridibundus were examined. Digeneans and nematodes were observed in both large-small intestines, lungs, rectum, mesenteries and organ surfaces; cestode and acanthocephalans were also observed in small intestine of $P$. ridibundus. There were $2.57 \pm 1.56$ ( $1-7$ with a range from) helminth species per infected host and there were 14.46 helminth individuals per infected host (except Abbreviata sp., uncountable). The helminth species that were observed in $P$. ridibundus, are common parasites of European anurans and also, some reptiles (e.g. E. colubrimurorum), aquatic birds (e.g. Eustrongylides sp.) fishes (e.g. P. laevis) (Yamaguthi, 1958, 1961, 1963; Liang-Sheng, 1958; Buchvarov, 1977; Yıldırımhan, 1999; Anderson, 2000; Dudiňák \& Šnábel, 2001; Düşen \& Öz, 2006, Yıldırımhan et al., 2007, Düşen et al., 2010a; Düşen, 2012). P. ridibundus represents a new host record for Nematotaenia dispar in Turkey.

The present study on the helminthfauna on P. ridibundus, Eurasian marsh frog in Denizli province (inner-west Anatolia region Turkey), offers a contribution to the present knowledge in this field.

\section{Acknowledgements}

This study was supported by TÜBİTAK (The Scientific and Technical Research Council of Turkey) Project no: 107T917, and partly supported by the Pamukkale University Scientific Research Projects Unit Project no: 2008BSP005. We also thank, for permission and helps, the Department of National Parks and Wildlife of the Ministry of Environment and Forestry, of the Republic of Turkey.

\section{References}

AL-SORKHY, M. K., AMR, Z. (2003): Helminth parasites of some amphibians in Jordan. Tr. J. of Zool., 27: 89 - 93

ANDERSON, R. C. (2000): Nematode Parasites of Vertebrates: Their Development and Transmission (2nd Edition). CABI Publishing, Wallingford, Oxon, U.K., 650 pp. AydoĞdu, A., Yildirimhan, H. S., Altunel, F. N. (1996a): An investigation on catfish (Silurus glanis L.) in Lake Iznik and its around, XIIIth National Biology Congress, 17 - 20 Eylül 1996. Cilt I, 63 - 70 (In Turkish) AydoĞdu, A., Yildirimhan, H. S., Altunel, F. N. (1996b): A Study on the parasites of Tench fishes of Lake Iznik (Tincta tincta L., 1758). Türkiye Parazitoloji Dergisi (Acta Parasitol. Tur.) 20: 261 - 270 (In Turkish, with English abstract)

AydoĞdu, A., Yildirimhan, H. S., Altunel, F. N. (1997): An investigation on the ecto and endoparasites of the Akbalık (Rutilus frisii), collected from Lake Iznik, IXth Aquaculture Congress, 17 - 19 Eylül 1997 Eğirdir/Isparta. 431 - 443 (In Turkish)

AydoĞdu, A., Yildirimhan, H. S., Altunel, F. N. (2000): The Helminth Fauna of the Adriatic Roach (Rutilis rubilio) in İznik Lake. Bull. Eur. Ass. Fish Pathol., 20 (3), 170

BAKER, M. R. (1987): Synopsis of the Nematoda parasitic in amphibians and reptiles. Memorial University of Newfoundland, Occas. Pap. in Biol., 1: 1 - 325

BARAN, I., ATATÜR, M. K. (1997): Turkish Herpetofauna. The Republic of Turkey, Ministry of Environment Publications, Ankara. 214 pp.

Bertman, M., OKUlEwicz, A. (1987): Lizards (Anguis fragilis L.) and snakes (Natrix natrix (L.)) as new hosts of Oswaldocruzia filiformis (Goeze, 1782) Travassos 1917 (Nematoda). Wiadomosci Parazytol., 33 (2): 209 - 212 (In Polish)

Biserkov, V., Kostadinova, A. (1998): Intestinal helminth communities in the green lizard, Lacerta viridis, from Bulgaria. J. Helminthol., 72 (3): 267 - 271. DOI: 10.1017/S0022149X00016540

BorkovCOVÁ, M., KopřIVA, J. (2005): Parasitic helminths of reptiles (Reptilia) South Moravia (Czech Republic). Parasitol. Res., 96: 77 - 78. DOI: 10.1007/s00436-0041258-6

BožKov, D., StojkovA, R. (1970): Beitrag zur untersuchung der Helminthenfauna der Rana graeca in Bulgarien. Bulletin de L'institute de Zoologie et Musée, Academie Bulgare des Sciences, 69 - 75 (In Bulgarian)

Buchvarov, G. K. (1977): Catalogue des helminthes des Amphibies en Bulgarie - Universite de Plovdiv "P Hilendarski”, Plovdiv, Bulgaria, 53 pp. (In Bulgarian).

Buchvarov, G. K., Petrov, P., Chochev, B. (1975): To the question about helminthofauna of Amphibious Eucaudate (Amphibia-Eucaudata) of Velingrad's District. Universite de Plovdiv "P Hilendarski”, Travaux Scientifiques, Biologie, 13 (4): 53 - 64

BudAK, A., GÖÇMEN, B. (2008): Herpetology (Herpetoloji) Ege Üniversity Press, Science Faculty Publ. no: 194 (2nd Edition) 226 pp. (In Turkish)

Cedhagen, T. (1988): Endoparasites in some Swedish amphibians. Acta Parasitol., 33: 107 - 113

DUDIŇÁK, V., ŠNÁBEL, V. (2001): Comparative Analysis of Slovak and Czech Populations of Pomphorhynchus laevis (Acanthocephala) Using Morphological and Isoenzyme Analyses. Acta Zool. Univ. Comenianae, 44: 41 - 50 DüŞEN, S. (2003): The helminth fauna of tailless frog species (Ordo: Anura) distributed in Antalya. PhD thesis, Akdeniz University, Antalya, Turkey. 116 pp. (in Turkish, with English abstract)

DüşEN S., ÖZ, M. (2004): Helminth parasites of the tree frog, Hyla arborea (Linnaeus, 1758) (Anura: Hylidae) from Southwest Turkey. Comp. Parasitol., 71 (2): 258 - 261. DOI: 10.1654/4123

DÜŞEN S., Öz, M. (2006): Parasitic helminths of the marsh frog, Rana ridibunda Pallas, 1771 (Anura: Ranidae), from Antalya Province, south-west Turkey. Comp. Parasitol., 73 (1): 121 - 129. DOI: $10.1654 / 4162.1$

DÜŞEN, S. (2007): Helminths of the two mountain frogs, Banded frog, Rana camerani Boulenger, 1886 and Uludağ Frog Rana macrocnemis Boulenger, 1885 (Anura: Ranidae), collected from Antalya Province. Acta Parasitol. Tur., 31 (1): $84-88$ 
DÜŞEN, S., OĞUZ, M. C. (2008): Occurence of Pomphorhynchus laevis (Acanthocephala) in the Marsh Frog (Rana ridibunda Pallas, 1771), from Turkey. Helminthologia, 45 (3): 154 - 156

Düşen, S., UĞUrTaş, İ. H., AydoĞDU, A., OĞUz, M. C. (2009): The helminth community of the Agile frog, Rana dalmatina Bonaparte, 1839 (Anura: Ranidae) Collected from Nortwest of Turkey. Helminthologia, 46 (3): 177 182. DOI: $10.2478 / \mathrm{s} 11687-009-0033-8$

Düşen, S., OĞUz, M. C., Barton, D., P. Aral, A., ŞulEKOĞLU, S., TePE, Y. (2010a): A metazoan parasitological research of three species of Anurans collected from the Çanakkale Province, Northwestern Turkey. North-West J. Zool., 6 (1): 25 - 35

DüŞEN, S., UĞURTAŞ, İ. H., AYdoĞDU, A. (2010b): Nematode parasites of the two limbless lizards: Turkish worm lizard, Blanus strauchi (Bedriaga, 1884) (Squamata: Amphisbaenidae), and slow worm, Anguis fragilis Linnaeus 1758 (Squamata: Anguidae), from Turkey. Helminthologia, 47 (3): 158 - 163. DOI: 10.2478/s11687-010-0024-9 DÜŞEN, S., OĞUZ, M. C. (2010): Metazoan endoparasites of three species of anurans collected from the Middle Black Sea region of Turkey. Helminthologia, 47 (4): 226 - 232. DOI: $10.2478 / \mathrm{s} 11687-010-0035-6$

DüşEN, S. (2011): The helminth parasites of the bufonid toads, European Common Toad, Bufo bufo (Linnaeus, 1758) and European Green toad, Bufo (Pseudepidalea) viridis Laurenti, 1768 (Anura: Bufonidae), collected from Denizli Province, Inner-West Anatolia Region, Turkey Helminthologia. 48 (2): $101-107$

DÜŞEN, S. (2012): First data on helminthfauna of the locally distributed a mountain frog, "Tavas Frog", Rana tavasensis Baran and Atatür, 1986 (Anura: Ranidae), from Inner-West Anatolia Region, Turkey. Tr J. of Zoology. 36(4): 496 - 502. DOI:10.3906/zoo-0909-15

FERNANDO, M. M. (1989): The parasitic burden of the frog Rana ridibunda Pallas, 1771 from Saudia Arabia. A preliminary list of parasitic helminths. Herpetol. J., 1: 415 - 417 GRABDA-KAZUBSKA, B., LEWIN, J. (1989): The helminth fauna of Bombina bombina (L.) and Bombina variegata (L.) in Poland. Acta Parasitol., 34: 273 - 279

HASSL, A. R (2010): A case of a facultative life-cycle diversification in the fluke Pleurogenoides sp. (Lecithodendriidae, Plagiorchiida). Wien Klin. Wochenschr. Suppl. 3: 91 - 93

HASsl, A. R., Richter, B., KÜBBERHEISS, A. (2010): Captive snake's care as parasite's life cycle conservation program. Herpetozoa., 23 (1/2): $83-85$

Heckmann, R. A., Oguz, M. C., Amin, O. M., Düşen, S., TEPE, Y., ASLAN, B. (2010): Host and geographical distribution of Pomphorhynchus spindletruncatus (Acanthocephala: Pomphorhynchidae) in Turkey, with enhanced description from new fish and amphibian hosts using SEM, and histopathological notes. Sci. Parasitol., 11: 129 - 139 Heckmann, R. A., Amin, O. M., Tepe, Y., Düşen, S., OGUZ, M. C. (2011): Acanthocephalus ranae (Acanthocephala: Echinorhynchidae) from amphibians in Turkey, with special reference to new morphological features revealed by SEM, and histopathology. Sci. Parasitol., 12: 23 - 32
Hristovski, N. D., Riggio, S. (1971): On some helminths from two toad species (Bufo bufo and B. viridis) collected in localities of Macedonia, Serbia (Yugoslavia), and Sicily (Italy). Fragmenta Balcanica Mus. Macedonica Sci. Nat., 8: $17-25$

Hristovski, N. D., LEES, E. (1973): The helminth fauna of Rana temporaria in relation to that of Europe generally. Acta Parasitol. Iugosl. 4: 93 - 98

Hristovski, N. D., Riggio, S. (1974): Some data on the parasitic helminthofaunas in populations of Hyla arborea Laur. From Yugoslavia, Greece and the island of Corsica. Acta Parasitol. Iugosl. 5: $21-23$

Hristovski, N., Smilkov, S., Tomovska, D., Popovich E., Kostich, D. (2006): Helmintofauna of Rana kurtmuelleri Gayda, 1940 syn. Rana balcanica Shneider et Sinish, 1992 (Anura:Ranidae) from Dojran Lake, Makedonia and Greece. $10^{\text {th }}$ International Congress on the Zoogeography and Ecology of Greece and Adjacent Regions Patras, Greece. June $26-30$.

IUCN (2012): Pelophylax ridibundus IUCN Red List of Threatened Species. Downloaded on 21 October 2012 from www.iucnredlist.org.

IBRAHIM, M. M. I. (2008): Helminth infracommunities of the maculated toad Amietophrynus regularis (Anura: Bufonidae) from Ismailia, Egypt. Dis. Aquat. Org., 82: 19- 26

KIRIN, D. (2002a): New data on the helminth fauna of Lacerta viridis Laurenti, 1768, and Podarcis muralis (Laurenti, 1768) (Reptilia: Lacertidae) in Bulgaria. Acta Zool. Bulg., 54 (1): $43-48$

KIR, İ., YILDIRIM, M. Z., BECER, A. AND İKIZ, R. (2001): The feeding habits and parasites of the lake frogs (Rana ridibunda Pallas, 1771; Anura: Ranidae) of Lake Eğirdir. Acta Parasitol. Tur., 25: $83-87$

KIRIN, D. (2002b): New records of the helminth fauna from grass snake, Natrix natrix L., 1758 and dice snake, Natrix tessellata Laurenti, 1768 (Colubridae: Reptilia) in South Bulgaria. Acta Zool. Bul., 54: 49 - 53

Kirin, D., Buchvarov, G. (2002): Biodiversity of of the helminth communities of acaudated Amphibians (Amphibia: Ecaudata) from Bistritsa Riverside (Gotse Delchev Region). Exp. Pathol. Parasitol., 5(8): $13-16$

KovalenKO, M. V. (2007): First Ukrainian record of the frog lung fluke Skrjabinoeces breviansa Sudarikov, 1950 (Trematoda: Plagiorchidae). Seriesbiology. Univ. Kharkov. no: 595.122(477). 93-96

KuC, I., SulgostowsKA, T. (1988a): Helminth fauna of Rana ridibunda Pallas, 1771 from Goclawski Canal in Warszaw (Poland). Acta Parasitol., 33: 101 - 105

Kuc, I., SulgostowsKA, T. (1988b): Helminth fauna of frogs in the forest of Kampinos near Warszawa. Acta Parasitol., 33: $267-272$

LIANG-SHENG, Y. (1958): A review of the trematode genus Encylometra Baylis and Cannon, 1924. J. Helminthol., 32: $99-144$

Masshait, N., Balouch, M., Mobedi, I. (2000): New records about helminth parasites of the marsh frog, Rana ridibunda (Anura: Ranidae), from the North of Iran. Iran. J. Fish. Sci., 2: $77-88$ 
MashaiI, N. (2005): Helminth Parasites of green toad, Bufo viridis (Anura: Bufonidae), Tree frog, Hyla arborea savignyi (Anura: Hylidae) and Marsh Frog, Rana ridibunda ridibunda (Anura: Ranidae) from Southwest of Iran. Iran. J. Vet. Res., 6 (3): $67-73$

MasshaiI, N., BAlOUCH M., Mobedi, I (2008): Report about helminth parasites of some Amphibians (Anura: Ranidae, Bufonidae) from the North and Northeast of Iran. J. Sci. Univ. Tehran., 33 (4): $9-13$

Mihalca, A. D., Gherman, C., Ghira, I., Cozma, V. (2007): Helminth parasites of reptiles (Reptilia) in Romania. Parasitol. Res., 95: 77 - 78. DOI: 10.1007/s00436007-0486-y

OĞUZ, M. C., Altunel, F. N., UĞurtaş, İ. H. (1994): An investigation of the species of Plathelminthes and Acanthocephalus ranae (Schrank, 1788, Acanthocephala) of marsh frogs (Rana ridibunda Pallas, 1771) which were collected from the Bursa and Edirne Regions. Turk J. Zool., 18: 47 - 51 (In Turkish, with English abstract)

ÖKTENER, A. (2003): A checklist of Metazoan Parasites Recorded in Freshwater Fish from Turkey. Zootaxa, 394: 1 $-28$

Prudhoe, S., A. Bray, S. (1982): Platyhelminth Parasites of Amphibia. British Museum of Natural History. Oxford University Pres, London. 217 pp

SAEED, I. E., AL-BARWARI S., AL-HARMNI, K. I. (2007): A metazoan parasitological research of some Iraqi Amphibians. Acta Parasitol. Tur., 3 (4): 337 - 345

SAĞLAM, N., ARIKAN, H. (2006): Endohelminth parasites of the Marsh Frog Rana ridibunda from Hazar Lake, Turkey. Dis. Aquat. Org., 72: 253 - 260

SANChis, V., Roig, J. M., CARretero, M. A., RocA, V., LlORENTE, G. A. (2000): Host - parasite relationships of Zootoca vivipara (Sauria: Lacertidae), in Pyreenes, North Spain. Folia Parasitol., 47: $118-122$

SAYGI, G., BAŞIBÜYÜK, H. H. (1990): Protozoon and metazoon parasites of Rana ridibunda. Acta Parasitol. Tur., 15: $105-118$

Schad, G. A., Kuntz, R. E., Wells, W. H. (1960): Nematode parasites from Turkish vertebrates: an annotated list. Can. J. Zoolog., 38: $949-963$

Sharpilo V. P., Biserkov, V., Kostadinova, A., BeHNKe, J. M., KUZMIN, Y. I. (2001): Helminths of the sand lizard, Lacerta agilis (Reptilia, Lacertidae), in the Palaearctic: faunal diversity and spatial patterns of variation in the composition and structure of component communities. Parasitology, 123: 389 - 400. DOI: 10.1017/S00 31182001008587

SHARPILO, V. P. (2003): Rare and locally distributed palearctic species of the reptile parasitic worms: Oxysomatium caucasicum (Nematoda, Cosmocertidae). Vesnik Zool., 37 (5): $69-72$

Shimalov, V. V., Shimalov, V. T. (2000): Helminth fauna of snakes (Reptilia, Serpentes) in Belorussian Polesye. Parasitol. Res., 86: 340 - 341. DOI: 10.1007/s004 360050055

Shimalov, V. T., Shimalov, V. V., Shimalov, A. V. (2000): Helminth fauna of lizards in the southern part of
Belarus. Parasitol. Res., 86: 343. DOI: 10.1007/s004360 050057

Shimalov, V. V., Shimalov V. T. (2001): Helminth fauna of toads in Belorussian Polesie. Parasitol. Res., 87: 84. DOI: $10.1007 / \mathrm{s} 004360000266$

Shimalov, V. V., Shimalov, V. T., Shimalov, A. V. (2001): Helminth fauna of newts in Belarusian Polesie. Parasitol. Res., 87: 356. DOI: 10.1007/PL00008592

TOPÇU, A., BAYRAK, N. (2000): The digestive system helminths of the Rana ridibunda 1771, Pallas and Bufo (Pseudepidalea) viridis Laurenti, 1768 (Anura) distributed in Nigde Province and its vicinity, XVth National Biology Congress, 5 - 9 Eylül 2000. Ankara. (In Turkish)

TOPÇU, A. (2002): The digestive system helminths found in the Taurus frog (Rana holtzi Werner, 1898), XVIth National Biology Congress, 4 - 7 Eylül 2002. Malatya. (In Turkish)

VAn Sluys, M., Schittini, G., Marra, R. V., AzeVedo, A., ViCENTE J. J., VRCIBRADIC, D., (2006): Body size, diet and endoparasites of the microhylid frog Chiasmocleis capixaba in an Atlantic Forest Area of Southern Bahiastate, Brazil. Brazil. J. Biol., 66 (1A): $167-173$

VASHETKO, E. V., SidDikov, B. H. (1999): The effect of the ecology of toads on the distribution of helminths. Turk. J. Zool., 23: $107-110$

Veith, M., ERPelding, G. (1995): Presence of Pomphorhynchus laevis in Salamandra salamandra. J Helminthol., 69: 267 - 268. DOI: 10.1017/S0022149X00014267

WALton, A. C. (1933): The nematoda as parasites of Amphibia. J. Parasitol. 20: (1) 1 - 33

YAmaguTI, S. (1958): Systema Helminthum. Vol. I: The Digenetic Trematodes of Vertebrates. Interscience Publishers, New York\& London 1575 pp

YamaguTI, S. (1961): Systema Helminthum: The nematodes of vertebrates. Vol III., Part II. Nematodes of Amphibians. Intersciences Publishers Ltd. London, England 679 pp YAMAGUTI, S. (1963): Systema Helminthum. Acanthocephala. Vol. V. Intersciences Publishers, London, England. $423 \mathrm{pp}$

Yildirimhan, H. S., UĞURTAŞ, İ. H., Altunel, F. N. (1996): An investigation on helminths of Rana ridibunda Pallas, 1771 (marsh frog). Acta Parasitol. Tur., 20: 113 130 (in Turkish, with English abstract)

YILDIRIMHAN, H. S., OĞUZ, M. C., UĞURTAŞ, İ. H. (1997a): An investigation on the nematodes of some tailless frogs (Rana ridibunda, Bufo bufo and Pelobates syriacus) collected from the Bursa regions. Hacettepe Fen ve Müh. Bil. Derg., 18: 45 - 58 (in Turkish with English abstract)

Yildirimhan, H. S., UĞURTaş I. H., Altunel, F. N. (1997b): An investigation on parasitic helminths of Rana macrocnemis Boulenger, 1885 (Uludag frog). Turk. J. Zool. 21: $467-473$

YILDIRIMHAN, H. S. (1999a): Researches on parasitic helminths of Bufo viridis Laurenti, 1768 (Anura: Amphibia). Turk. J. Zool, 23: $177-195$

YILDIRIMHAN, H. S. (1999b): Helminth fauna of lizards species belonging to Lacertidae (Reptilia) family distributed in Bursa and its around (PhD thesis). Uludağ Univer- 
sity, Institute of Science. 120 pp, Bursa (In Turkish, with English abstract)

YIldirimHAN, H. S., AYdOĞDU, A., UĞURTAŞ, I. H., Altunel, F. N. (2001a): Helminth fauna of Bombina bombina (Linnaeus, 1767) (Fire-Bellied Toad) collected from Sakarya and Edirne (Turkey). Acta Parasitol. Tur., 25 (3): 308 - 311 (in Turkish, with English abstract)

YILDIRIMHAN, H. S., AYDOĞDU, A., UĞURTAŞ, I. H., AltUNEL, F. N. (2001b): An investigation on Plathelminth and Acanthocephala of Mertensiella caucasica (Caucasian Salamander). Acta Parasitol. Tur., 25: 393 - 397 (In Turkish, with English abstract)

YILDIRIMHAN, H. S., KARADENIZ, E., GÜRKAN, E., KoyUn, M. (2005a): Metazoon parasites of the Marsh frog (Rana ridibunda Pallas, 1771; Anura) collected from the different regions in Turkey. Acta Parasitol. Tur., 29 (2): 135 - 139 (In Turkish with English abstract)

Yildirimhan, H. S., Bursey C. R., GoldberG, S. R. (2005b): Helminth parasites of the Caucasian salamander, Mertensiella caucasica, from Turkey. Comp. Parasitol., 72(1): 75 - 87. DOI: 10.1654/4152

Yildirimhan, H. S., Goldberg S. R., Bursey, C. R. (2006a): Helminth parasites of the Banded Frog, Rana camerani (Ranidae) from Turkey. Comp. Parasitol., 73(2): 222 - 236. DOI: 10.1654/4229.1

Yildirimhan, H. S., Bursey, C. R. GoldberG, S. R. (2006b): Helminth parasites of the Taurus frog, Rana holtzi, and the Uludag frog, Rana macrocnemis, with remarks on the helminth community of Turkish anurans. Comp. Parasitol., 73 (2): 237 - 248. DOI: 10.1654/4191.1 Yildirimhan, H. S., Altunel F. C., UĞURTAŞ, I. H. (2006c): Helminth parasites of Hyla arborea (Linneaus, 1758) (Tree Frog) collected from Bursa, Edirne and Sa- karya. Acta Parasitol. Tur., 30 (1): 56 - 59 (In Turkish with English abstract)

Yildirimhan, H. S., KARADENIZ, E. (2007): Helminth Parasites of the Common Toad, Bufo bufo (Linnaeus, 1758) (Anura: Bufonidae) from Northeast Turkey. Comp. Parasitol., 74 (1): 176 - 178. DOI: 10.1654/4246.1

YILDIRIMHAN, H. S. (2008): A Preliminary investigation on helminth fauna of Triturus vittatus (Jenyns, 1835) and $T$. karelinii (Strauch, 1870). Acta Parasitol. Tur., 32 (2): 158 - 160 (In Turkish with English abstract)

Yildirimhan, H. S., Bursey, C. R. GoldberG, S. R. (2009): Helminth Parasites of the Caucasian Parsley Frog, Pelodytes caucasicus, from Turkey. Comp. Parasitol., 76(2): 247 - 257. DOI: 10.1654/4376.1

YILDIRIMHAN, H. S., BURSEY, C. R. (2010): Helminth parasites of the eastern spadefoot toad, Pelobates syriacus (Pelobatidae), from Turkey. Turk. J. Zool., 34: 11 - 319

Yildirimhan, H. S., Bursey, C. R., Altunel, F. N. (2011): Helminth parasites of the Balkan green lizard, Lacerta trilineata Bedriaga 1886, from Bursa, Turkey. Turk. J. Zool., 35(4): $519-535$

Yildirimhan, H. S., NuRHAN, S., İNCEDOĞAN, S., BuRSEY, C. R. (2012): Helminth parasites of the lemonyellow tree Anura and Urodela, Hyla savignyi (Hylidae), from Turkey. Turk. J. Zool., 36: $171-184$

YILDIZ, K. (2003): Helminth Infections in Tench (Tinca tinca) from Kapulukaya Dam Lake. Turk. J. Vet. Anim. Sci., 27: 671 - 675 (In Turkish)

Ziolkowska, M., RoKicki, J. (2003): An Attempt to Determine the Intermediate Host for Pomphorhynchus laevis (Acanthocephala) in the Baltic Sea. Acta Ichtyol. Pisc., 37 $-43$

ACCEPTED DECEMBER 4, 2012 